\title{
Small Atomic Displacements Recorded in Bismuth by the Optical Reflectivity of Femtosecond Laser-Pulse Excitations
}

\author{
D. Boschetto, ${ }^{1, *}$ E. G. Gamaly, ${ }^{2}$ A. V. Rode, ${ }^{2}$ B. Luther-Davies, ${ }^{2}$ D. Glijer, ${ }^{1}$ T. Garl, ${ }^{1}$ O. Albert, ${ }^{1}$ \\ A. Rousse, ${ }^{1}$ and J. Etchepare ${ }^{1}$ \\ ${ }^{1}$ Laboratoire d'Optique Appliquée, ENSTA/Ecole Polytechnique, Palaiseau, France \\ ${ }^{2}$ Laser Physics Centre, Research School of Physical Sciences and Engineering, The Australian National University, \\ Canberra, ACT 0200 Australia
}

(Received 27 February 2007; published 18 January 2008)

\begin{abstract}
Subtle atomic motion in a Bi crystal excited by a 35 fs-laser pulse has been recovered from the transient reflectivity of an optical probe measured with an accuracy of $10^{-5}$. Analysis shows that a novel effect reported here - an initial negative drop in reflectivity - relates to a delicate coherent displacement of atoms by the polarization force during the pulse. We also show that reflectivity oscillations with a frequency coinciding with that of cold $\mathrm{Bi}$ are related to optical phonons excited by the electron temperature gradient through electron-phonon coupling.
\end{abstract}

DOI: 10.1103/PhysRevLett.100.027404

PACS numbers: 78.47.-p, 63.20.-e, 78.68.+m

Studies of femtosecond laser-solid interactions where the pulse duration is shorter than the period of phonon oscillations as well as the major relaxation times present an exciting field of research in modern solid-state physics [113]. Such a short pulse excites only the electron subsystem while the lattice remains cold during the electron-phonon energy transfer time. There is a quest for information on the atomic motion driven by the excited electrons in nonequilibrium conditions, which would uncover the microscopic nature of material transformation on a femtosecond time scale and on a nanometer spatial scale. Since the early 1980s a number of pump-probe experiments have been performed which measured the transient reflectivity and transmission of solids. The most salient feature of those measurements was reflectivity oscillations at frequencies corresponding to the optical phonon modes of the material. A number of mechanisms have been proposed to explain those oscillations (for example, displacive excitation of coherent phonons [2] and impulsive stimulated Raman scattering [3,4]). However, a general theory explaining excitation of atomic vibrations in transparent and opaque media and their connection to reflectivity oscillations is still absent, to the best of our knowledge.

In this Letter we present experimental and theoretical studies of reflectivity oscillations of an optical probe beam reflected from a single crystal of bismuth excited to temperature exceeding that of the melting. The interconnected processes of electron heating by laser absorption, electronelectron, electron-phonon, and phonon-phonon interactions are considered. We show that atomic vibrations at the "cold" phonon frequency are excited by the electron temperature gradient during the electron-to-phonon energy transfer time. The resulting fast atomic motion affects the transient dielectric function and reflectivity via electronphonon coupling and polarization. The theory allows recovery of the main microscopic atomic motion from the experiments for times from femtoseconds to tens of picoseconds.

The experiment was carried out in a standard pumpprobe geometry by a Ti:sapphire laser system that could deliver $35 \mathrm{fs}$ pulses at a $1 \mathrm{kHz}$ repetition rate at $800 \mathrm{~nm}$ wavelength and pulse energy up to $10 \mathrm{~mJ}$. The pump pulse was chopped at $500 \mathrm{~Hz}$, and the probe reflectivity was measured using a digital lock-in amplifier. Our signal-tonoise ratio was $10^{5}$, allowing us to observe reflectivity changes $\Delta R$ with the accuracy $\Delta R / R \sim 10^{-5}$. The temporal resolution was determined by the probe pulse duration and was $35 \mathrm{fs}$. The pump and probe pulses were focused to beams $100 \mu \mathrm{m}$ in diameter on the sample, which was a single crystal of bismuth (111)-oriented with one side polished to reduce light scattering at the surface.

Figure 1 shows the time-dependent reflectivity for two pump fluencies: $2.7 \mathrm{~mJ} / \mathrm{cm}^{2}$ and $6.7 \mathrm{~mJ} / \mathrm{cm}^{2}$. These traces contain characteristic features that were observed at all pump fluences in the range $1.5 \mathrm{~mJ} / \mathrm{cm}^{2}$ to $15 \mathrm{~mJ} / \mathrm{cm}^{2}$. For an instant the reflectivity drops below the unperturbed value before increasing above the initial level reaching a maximum about $300 \mathrm{fs}$ after the excitation pulse. The reflectivity then oscillates with a fluencedependent frequency and with amplitude, which decays nearly exponentially with time. About $10 \mathrm{ps}$ after the excitation pulse the signal again drops below the unperturbed level and remains constant and below this level for $\sim 20 \mathrm{ps}$, before slowly returning to the unperturbed value $\sim 4$ ns after excitation. This indicated that material excitation was fully reversible. The frequency of the reflectivity oscillations for a pump fluence of $2.7 \mathrm{~mJ} / \mathrm{cm}^{2}$ coincided with the value of the $A_{1 g}$ optical mode of bismuth at $2.9 \mathrm{THz}$. The frequency decreased with increasing pump fluence (at $6.7 \mathrm{~mJ} / \mathrm{cm}^{2}$ it was $2.86 \mathrm{THz}$ ) in agreement with previous experiments [6]. A novel feature observed in these experiments was the presence of the initial sharp 

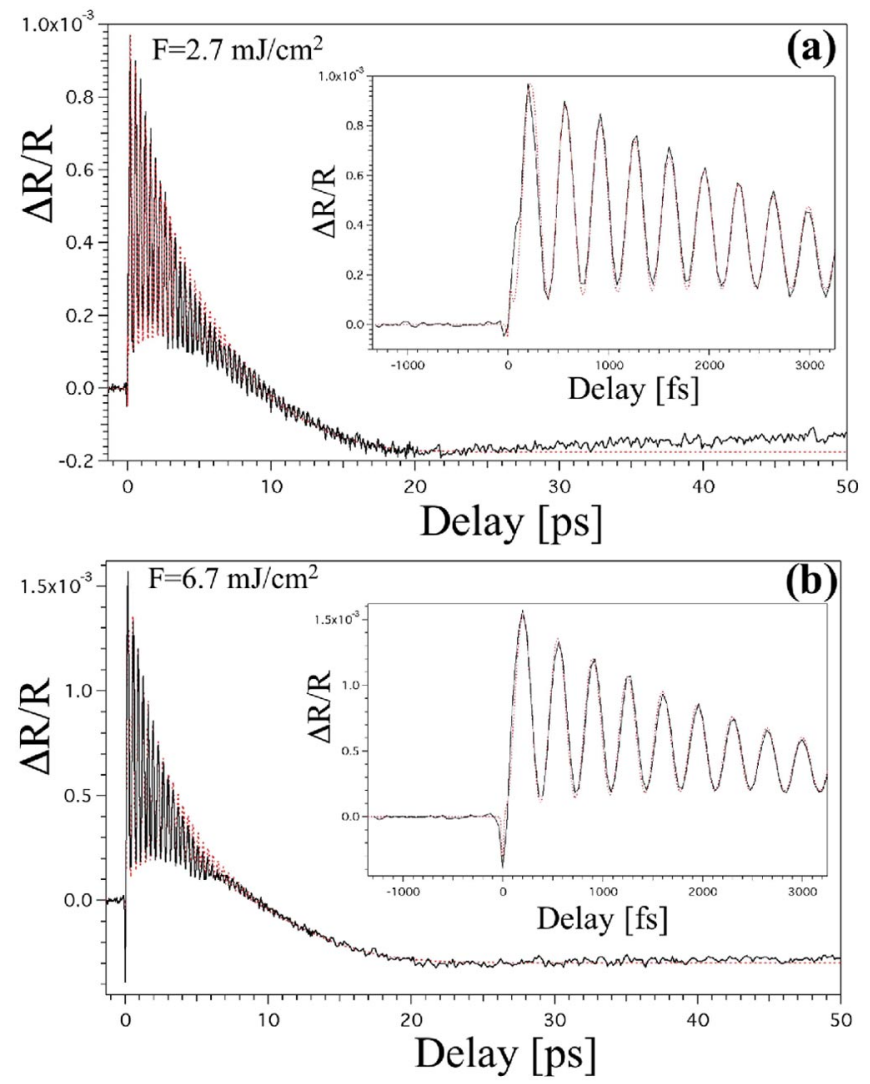

FIG. 1 (color online). Comparison between the experimental [solid (dark) line] and theoretical [dashed (red) line] behavior of the transient reflectivity: (a) excitation flux $2.7 \mathrm{~mJ} / \mathrm{cm}^{2}$ and (b) excitation flux $6.7 \mathrm{~mJ} / \mathrm{cm}^{2}$.

drop of the reflectivity, which we could observe only when the time resolution was better than $40 \mathrm{fs}$.

Reflectivity oscillations are often related solely to the coupling of the laser field to the field associated with atomic vibrations, i.e., the impulsive Raman effect [3$6,14]$. However, there are a variety of other important processes during the laser-matter interaction that affect the material properties. We analyze them below in succession starting from the arrival of the laser pulse.

The laser pulse is shorter than the electron-phonon energy transfer time. Therefore only electrons are heated during the pulse [15]. The electron energy density absorbed in the skin layer, $E_{e l}=C_{e} n_{e} T_{e}=2 A F\left(t_{p}\right) / l_{s}$, reaches a maximum at the end of the pulse. Here $A=$ $1-R=0.26$ [16-18] is the absorption coefficient, $F\left(t_{p}\right)$ is the laser fluence, $t_{p}$ is laser pulse duration, $n_{e}$ and $C_{e}$ are electron number density and heat capacity, respectively, $T_{e}$ is the electron's temperature, and $l_{s}=2.984 \times 10^{-6} \mathrm{~cm}$ is the skin depth. The absorbed energy density equals $(0.48-1.19) \times 10^{3} \mathrm{~J} / \mathrm{cm}^{3}$ (for $2.7 \mathrm{~mJ} / \mathrm{cm}^{2}-6.7 \mathrm{~mJ} / \mathrm{cm}^{2}$ ) and is comparable to the enthalpy of melting in equilibrium [17]. The number density of electrons excited to the conduction band by an avalanchelike process is proportional to the electron energy density: $n_{e}\left(t_{p}\right) \cong\left[2 A F\left(t_{p}\right)\right] /\left(\Delta E l_{s}\right)$ (here $\Delta E$ is the energy required for a transition from the valence to the conduction band). The electron number density at the end of the pump pulse is proportional to the maximum electron temperature $T_{e, \max }\left(t_{p}\right)$. We assume that at $t>t_{p}$ the above proportionality also holds, $\Delta n_{e} / n_{e} \propto T_{e}(t) / T_{e, \max }$, because the recombination rate for $\mathrm{Bi}$ is unknown, to the best of our knowledge.

Electron-electron collisions quickly lead to the establishment of a local equilibrium. Indeed, the electronelectron collision rate for strongly correlated degenerate electron gas reads [19] $\nu_{e e} \propto \omega_{p} \varepsilon_{e} / \varepsilon_{F}$ ( $\varepsilon_{e}$ is the electron energy in excess over the Fermi level, $\varepsilon_{F}$, and $\omega_{p}$ is plasma frequency). For the conditions of the experiment, $\varepsilon_{e} \cong$ $0.02 \varepsilon_{F}$, and $\omega_{p e}=1.3 \times 10^{16} \mathrm{~s}^{-1}\left(\omega_{p e}\right.$ and $\varepsilon_{F}$ are extracted from the optical data [16-18]). Thus an equilibrium distribution for electrons is established in a period, $t_{e e} \approx$ $\nu_{e e}^{-1} \sim 4$ fs.

Phonon-phonon interactions establish equilibrium in the lattice. The effective phonon-phonon collision rate reads $[20,21] \nu_{\mathrm{ph}-\mathrm{ph}} \cong \omega_{D} T_{L} / T_{D}\left(\omega_{D}, T_{D}\right.$, and $T_{L}$ are, respectively, the Debye frequency, the Debye, and lattice temperature). The phonon-phonon relaxation time in our case $\left(\omega_{D}=1.56 \times 10^{13} \mathrm{~s}^{-1} ; T_{D}=119 \mathrm{~K}\right)$ lies in the range $t_{\mathrm{ph}-\mathrm{ph}}=\left(\nu_{\mathrm{ph}-\mathrm{ph}}\right)^{-1} \sim 8-80 \mathrm{fs}$. The heating time for the lattice is determined by the electron-phonon energy transfer rate [21-23], $t_{e \text {-ph }}^{\text {energy }}=\left(\nu_{e \text {-ph }}^{\text {energy }}\right)^{-1} \approx\left(\hbar \omega_{D}^{2} T_{L}^{2} / T_{D}^{2} \varepsilon_{F}\right)^{-1}$. In the conditions of our experiments $t_{e \text {-ph }}^{\text {energ }}$ varies in the range 5-20 ps. The time for cooling the skin depth of $29.84 \mathrm{~nm}$ is $t_{\text {cool }} \approx l_{s}^{2} / D=133$ ps (diffusion coefficient in Bi $D=0.067 \mathrm{~cm}^{2} / \mathrm{s}$ [17]). It is 4 times longer than the observation period ( $\sim 25 \mathrm{ps})$ in our experiments, and therefore heat losses can be neglected.

The above relation between relaxation times is the rationale for using the 2-temperature approximation $\left[T_{e}(t), T_{L}(t)\right]$ to describe the nonequilibrium laser-matter interaction [22,23]. We obtained $T_{e}(t), T_{L}(t)$ from numerical solutions of coupled electron and lattice energy equations $[22,23]$ with the material data taken from [16-18]. The maximum electron temperature (average energy per particle) at the end of the pulse is found to be $T_{e, \max }=$ $\left[4 \varepsilon_{F} A F\left(t_{p}\right) / \pi^{2} n_{e} l_{s}\right]^{1 / 2}, T_{e, \max }=2825 \mathrm{~K}-4450 \mathrm{~K}$ for fluences $2.7 \mathrm{~mJ} / \mathrm{cm}^{2}$ and $6.7 \mathrm{~mJ} / \mathrm{cm}^{2}$, respectively. The maximum lattice temperature is reached after equilibration of electron and lattice temperatures after $\sim 20 \mathrm{ps,} \mathrm{and} \mathrm{is,}$ correspondingly, $701.5 \mathrm{~K}$ and $1273 \mathrm{~K}$. The real part of the complex dielectric function can be considered as the sum of a small positive term due to the polarization change and a negative electronic term along with the electronic imaginary part, responsible for absorption, $\varepsilon=\varepsilon_{\mathrm{pol}}+\varepsilon_{e, r}+$ $i \varepsilon_{e, i}$. Optical data can be described with good accuracy by the Drude model with only the electronic terms [1618]. The real and imaginary electronic parts are [24] $\varepsilon_{r}=$ $1-\omega_{p}^{2} /\left(\omega^{2}+\nu_{e-\mathrm{ph}}^{2}\right) ; \varepsilon_{i}=\left(1-\varepsilon_{r}\right) \nu_{e-\mathrm{ph}} / \omega$. Here $\omega_{p}^{2}=$ $4 \pi e^{2} n_{e}(t) / m_{e}, \omega$, and $\nu_{e-\mathrm{ph}}$ are, respectively, the plasma 
frequency square, the laser frequency, and the electronphonon momentum exchange rate. Then, the change in the electronic dielectric function due to laser excitation reads

$$
\Delta \varepsilon_{r, i}=\left(\partial \varepsilon_{r, i} / \partial n_{e}\right) \Delta n_{e}+\left(\partial \varepsilon_{r, i} / \partial \nu_{e-\mathrm{ph}}\right) \Delta \nu_{e-\mathrm{ph}} .
$$

The electron-phonon momentum exchange rate is proportional to phonon amplitude, $q$ [25]: $\nu_{e-\mathrm{ph}} \approx n_{\mathrm{ph}} q^{2} v_{e}-$ in agreement with quantum [21] and kinetic [27] calculations; $v_{e}$ is the electron velocity, $n_{\mathrm{ph}} \approx n_{a} T_{L}(t) / T_{D}$ is the number density of phonons, and $n_{a}$ is the atomic number density. The perturbation in the coupling rate is written assuming that the change in the electron velocity in comparison to the Fermi velocity is negligible: $\Delta \nu_{e-\mathrm{ph}} / \nu_{e \text {-ph }}^{(0)}=$ $\Delta T_{L} / T_{0}+2 \Delta q(t) / q_{0}$. Here zero denotes initial room temperature and the phonon amplitude, $\Delta q \ll q_{0}$. The coefficients in (1) are calculated using the unperturbed optical data from [16-18].

The change of dielectric function due to polarization in accordance with the Plazcek model is proportional to the atomic displacement induced by the laser field [14,21], $\Delta \varepsilon_{\text {pol }}^{i i} \approx 4 \pi\left(\frac{\partial \alpha^{i i}}{\partial x_{k}}\right)_{0} x_{k} \approx \frac{4 \pi \alpha_{0}^{i i} q_{k}}{d_{0}}\left(\alpha_{0} \sim 0.2\right.$ is the zero order polarizability, and $d_{0}$ is the interatomic distance). The forces exerted on atoms by the laser are of two types. The polarization force expressed through the above change in the dielectric function as follows [14,21]: $f_{k}^{\mathrm{pol}}=$ $\frac{\partial\left(\Delta \varepsilon_{\text {pol }}^{i i}\right)}{\partial x_{k}} \frac{E_{i} E_{i}}{8 \pi n_{a}} \approx 4 \pi\left(\frac{\partial \alpha^{i i}}{\partial x_{k}}\right)_{0} \frac{E^{2}}{2 n_{a}}$, where $E$ is the laser electric field. In conditions of the experiments the pump beam was incident along the normal ( $z$ direction). It was linearly polarized along the surface ( $x$ direction). This force is proportional to the laser intensity $f_{x}^{\mathrm{pol}} \approx 4 \pi \alpha_{0}^{x x} I / c n_{a} d_{0}$ [3,14], and its magnitude is estimated to be $\sim 10^{-7}$ dyne. One can estimate the atomic shift, $\Delta q$, under the action of this force during the pulse, $t_{p}, \Delta q_{x} \approx f_{x}^{\mathrm{pol}} t_{p}^{2} / 2 M$, where $M$ is the atomic mass, and corresponding positive change in the dielectric function $\Delta \varepsilon_{\mathrm{pol}}^{x x} \sim 10^{-5}$.

During the pulse the electrons are rapidly and nonuniformly heated while the lattice remains cold. The time and space dependence of the electron temperature during the pulse reads, $T_{e}(z, t)=T_{e, \max }\left(t / t_{p}\right)^{1 / 2} \exp \left(-z / l_{s}\right)$. The electrostatic field that is proportional to the gradient of the electron temperature drives atomic motion. This electronic force is directed along the normal to the target surface (along the evanescent laser field) as follows: $f_{z}^{\text {elec }}=-\nabla_{z} T_{e}=\left(T_{e, \max } / l_{s}\right)\left(t / t_{p}\right)^{1 / 2} \exp \left(-z / l_{s}\right)$.

For the first $10 \mathrm{fs}$ of the pulse the polarization force dominates, and then the growing electronic force begins to prevail reaching a maximum value (twice the polarization force) at the end of the pulse. The total thermal force includes components due to both the (decreasing) electronic temperature and the (increasing) lattice temperature and acts in the $z$ direction displacing the atoms, $f_{z}^{\text {th }}=$ $\left[T_{e}(t)+T_{L}(t)\right] / l_{s}$. However, for excitation of the cold phonon mode the electrons' temperature gradient domi- nates. One can describe the vibrations as an approximate solution of the equation for damped harmonic oscillations [3,14]: $\Delta q_{z}(t) \approx\left\{f_{z}^{\text {th }}(t) / M \omega_{0} \gamma\right\} \cdot e^{-\gamma t} \cos \left\{\left(\omega_{0}^{2}-\right.\right.$ $\left.\left.\gamma^{2}\right)^{1 / 2} t+\varphi\right\}$, where $\omega_{0}$ is $A_{1 g}$ phonon frequency, $\gamma$ and $\varphi$ are the phenomenological damping due to phononphonon coupling and phase shift, respectively, and $\Delta q_{z} \ll$ $q_{0}$. The reflectivity variations of a probe are expressed through the changes in the dielectric function, which in turn are explicit functions of time-dependent electron and lattice temperatures. Indeed, $\Delta R=\left(\partial R / \partial \varepsilon_{r}\right)_{0} \Delta \varepsilon_{\text {pol }}+$ $\left(\partial R / \partial \varepsilon_{r}\right)_{0} \Delta \varepsilon_{e, r}+\left(\partial R / \partial \varepsilon_{i}\right)_{0} \Delta \varepsilon_{e, i}$. Here the coefficients are determined from the unperturbed values of the dielectric function for $\mathrm{Bi}$ at $800 \mathrm{~nm}, \varepsilon_{r}=-16.25, \varepsilon_{i}=15.4$; $R=0.74[14,17,18]$ by the Fresnel formula [24]. It follows that for $\mathrm{Bi}$ at $800 \mathrm{~nm},\left(\frac{\partial R}{\partial \varepsilon_{r}}\right)_{0}<0$ and $\left(\frac{\partial R}{\partial \varepsilon_{i}}\right)_{0}<0$, which is typical for good metals. Finally, the transient reflectivity is expressed through the time-dependent electron and lattice temperatures as follows:

$$
\begin{aligned}
\Delta R= & -C_{1} \Delta \varepsilon_{\mathrm{pol}}+C_{2} T_{e}(t) / T_{e, \max }-C_{3} T_{L}(t) / T_{e, \max } \\
& -C_{4} \Delta q_{z}(t) / q_{0}
\end{aligned}
$$

The signs of normalization coefficients, $C_{i}$, are defined by the unperturbed optical properties of the Bi crystal [28]. The first term in (2) is responsible for the polarization during the pulse due to the impulsive Raman effect. The second term emphasizes the increase in reflectivity due to electron excitation from the valence to the conduction band. The third term is responsible for the lattice heating, while the fourth term emphasizes polarization by the thermal force during the pulse and the atomic vibrations afterward. It follows from (2) that during the pulse the first, second, and fourth terms with the effects of opposite sign compete. The polarization components switch on from the very beginning while the number of free carriers, that is, proportional to the electron temperature, grows up during the pulse. Therefore the first negative initial drop in reflectivity should be always present early in the pulse until the second term in (2) becomes dominant. The amplitude of the initial drop is proportional to the laser intensity in agreement with the experiments. The reflectivity reaches its maximum after approximately one phonon period due to electron excitation and heating. Afterward phonons oscillations become apparent in the time dependence of the reflectivity.

The calculated $\Delta R / R$ function is presented in Fig. 1 and compared with the experimental data. There are several distinguishable features in the reflectivity behavior. First, the sharp initial drop in reflectivity was observed experimentally with a 35 fs pump probe, while with lower temporal resolution of $50 \mathrm{fs}$ it was not detected that qualitatively agrees with the theory. Second, after reaching maximum, the reflectivity starts oscillating with the period corresponding to the $A_{1 g}$ phonon frequency, $2.9 \mathrm{THz}$ at laser fluence $2.7 \mathrm{~mJ} / \mathrm{cm}^{2}$, and $2.86 \mathrm{THz}$ at $6.7 \mathrm{~mJ} / \mathrm{cm}^{2}$ 
that is the mode softening observed before [6]. The damping of the oscillations, with $\gamma=3.4 \times 10^{11} \mathrm{~s}^{-1}$, results from multiphonon processes (anharmonicity) that finally terminate harmonic vibrations due to lattice temperature increase up to the melting point. Note that the duration of the period where harmonic oscillations are discernible decreases with the increase in the pump fluence in qualitative agreement with the above considerations. Third, the average value of the oscillating reflectivity drops below the unperturbed level after approximately $10 \mathrm{ps}$ due to free carrier recombination and electron-to-lattice temperature equilibration. Theoretical predictions based on Eq. (2) fit well to experiments during the first 25 ps. The slight discrepancy for a longer period most probably relates to the heat diffusion from the skin layer, which was not included in the calculations.

To conclude, we demonstrated that the reflectivity oscillations measured with high temporal resolution allow recovery of the subtleties of atomic motion in a fs-laser excited solid during the period from several femtoseconds to tens of picoseconds. Atomic motion begins with a slight coherent displacement of atoms that produces a small drop in reflectivity. An impulse due to electron excitation by the laser pulse excites harmonic oscillations of the practically cold lattice, and these are imprinted into the reflectivity through electron-phonon coupling. The atomic motion becomes anharmonic at a stage when lattice temperature approaches to that for equilibrium melting and the reflectivity oscillations disappear. General relations between forces exerted on atoms during laser-matter interaction, atomic motion, and optical parameters have been established.

E. G. G., A. V.R., and B.L.D. gratefully acknowledge the support of the Australian Research Council through its Discovery and Federation programs. The support of Programme International De Cooperation Scientifique (PICS, France) as well as the European contract FLASH (No. MRTN-CT-2003-503641) is gratefully acknowledged. The authors thank L. Antonucci and G. Rey for their help with the laser system.

*davide.boschetto@ensta.fr

[1] A. Rousse et al., Nature (London) 410, 65 (2001).

[2] H. J. Zeiger et al., Phys. Rev. B 45, 768 (1992).

[3] R. Merlin, Solid State Commun. 102, 207 (1997).
[4] G. A. Garrett et al., Phys. Rev. Lett. 77, 3661 (1996).

[5] M. Hase et al., Appl. Phys. Lett. 69, 2474 (1996).

[6] E. D. Murray et al., Phys. Rev. B 72, 060301(R) (2005), and references therein.

[7] K. Ishioka et al., J. Appl. Phys. 100, 093501 (2006).

[8] K. Sokolowski-Tinten et al., Nature (London) 422, 287 (2003).

[9] A. Cavalleri et al., Phys. Rev. Lett. 87, 237401 (2001).

[10] E. Collet et al., Science 300, 612 (2003).

[11] T. Dumitrica et al., Phys. Rev. Lett. 92, 117401 (2004).

[12] M. Chollet et al., Science 307, 86 (2005).

[13] A. J. Kent et al., Phys. Rev. Lett. 96, 215504 (2006).

[14] Y. R. Shen and N. Bloembergen, Phys. Rev. 137, A1787 (1965).

[15] E. G. Gamaly et al., Phys. Plasmas 9, 949 (2002).

[16] T. Garl (private communication).

[17] American Institute of Physics Handbook, edited by D. E. Gray (McGraw-Hill, New York, 1972), 3rd ed.

[18] Numerical Data and Functional Relationships in Science and Technology, edited by O. Madelung, M. Schulz, and H. Weiss, Landolt-Börnstein, New Series, Group III, Vol. 17 (Springer-Verlag, Berlin, 1983).

[19] David Pines, Elementary Excitations in Solids (W. A. Benjamin, New York, 1964).

[20] E. M. Lifshitz and L.P. Pitaevskii, Physical Kinetics (Pergamon Press, Oxford, 1981).

[21] Yu. A. Il'inskii and L. V. Keldysh, Electromagnetic Response of Material Media (Plenum Press, New York, 1994).

[22] M. I. Kaganov et al., Sov. Phys. JETP 4, 173 (1957).

[23] P. B. Allen, Phys. Rev. Lett. 59, 1460 (1987).

[24] L. D. Landau, E. M. Lifshitz, and L.P. Pitaevskii, Electrodynamics of Continuous Media (Pergamon Press, Oxford, 1984).

[25] Electron-phonon coupling represents the interaction between the electron charge, $e$, and the dipole electric field of polarized charge created by lattice vibrations, $E_{\mathrm{ph}} \approx$ $e q / d_{0}^{3}$ [26]. The electron-phonon momentum exchange rate expresses through the energy of interaction, $\varepsilon_{\mathrm{ph}} \approx$ $e q \mathrm{E}_{\mathrm{ph}} \approx e^{2} q^{2} / d_{0}^{3}$, in agreement with the quantum approach [21]: $\nu_{e-\mathrm{ph}} \approx \varepsilon_{\mathrm{ph}} / \hbar \approx n_{\mathrm{ph}} q^{2} v_{e} ; v_{e} \approx e^{2} / \hbar$ is the electron velocity and $n_{\mathrm{ph}} \approx d_{0}^{-3}$ is the phonon's density.

[26] A. A. Abrikosov, Sov. Phys. JETP 17, 1372 (1963).

[27] M. Ziman, Electrons and Phonons (Clarendon Press, Oxford, 1960).

[28] $C_{1} \sim A_{1}=\left(\frac{\partial R}{\partial \varepsilon_{r}}\right)_{0}<0 ; \quad C_{2} \sim A_{2}=\left(\varepsilon_{r, m}-1\right)\left(\frac{\partial R}{\partial \varepsilon_{r, m}}\right)_{0}+$ $\varepsilon_{i, m}\left(\frac{\partial R}{\partial \varepsilon_{i, m}}\right)_{0}>0 ; C_{3}, C_{4} \sim A_{3}=\frac{2 \varepsilon_{i} \omega \nu}{\omega^{2}+\nu^{2}}\left(\frac{\partial R}{\partial \varepsilon_{e, r}}\right)_{0}+\varepsilon_{e, i} \frac{\left(\omega^{2}-\nu^{2}\right)}{\left(\omega^{2}+\nu^{2}\right)} \times$ $\left(\frac{\partial R}{\partial \varepsilon_{e, i}}\right)_{0}<0$. Here $\nu$ is electron-phonon momentum exchange rate and $\omega$ is the laser frequency. 